\title{
Image Compressing Method for Checked Textile Designs by Using Distributions in the Spatial Frequency Domains
}

\author{
Ken'ichi Ohta*, Toshihiko Tanaka and Fujio Miyawaki \\ Himeji Institute of Technology, Shosha 2167, Himeji, Hyogo, 671-2201, Japan
}

Received 17 August 1999; Accepted 21 February 2000

\begin{abstract}
Checked textile designs have many repeated patterns, so their distributions in the spatial frequency domains are partially concentrated. In addition, each distribution around local values in the spatial frequency domains can be approximately regarded as the normal distribution. By this property, it is possible to reconstruct distributions close to original distributions by using characteristic values of each distribution around local values. Moreover, in checked images, it is possible to obtain reconstructed images close to original images by using the data on the vertical and horizontal axes in the spatial frequency domains. So, we focused on reconstructing only distributions on the vertical and horizontal axes in the spatial frequency domains to compress checked images and used Gram-Charlier approximate equation to reconstruct the distributions. In this method, the mean, valiance, skewness and kurtosis [1-3] were used as characteristic values of the distributive shapes. Finally, we used only local values and these four data of distributive shapes around the local values as the compressed codes, and the other data aren't required. We propose the compressing method using these properties and show the effectiveness for checked images.
\end{abstract}

Keywords: The fast Fourier transform, checked textile design, distributions in spatial frequency domains, local values, image compression

\section{Introduction}

When constructing a database of images, a vast amount of data is generally required. Therefore, in order to reduce the overload when images are stored, many image-compressing methods have been suggested. Now influential image compressing method is JPEG. Almost images on net are compressed by JPEG or GIF format. However, in these methods, the compression ratio is not dramatically high, and there are some faults. They are block noise; mosquito noise, and so on. In such an image-compressing field, the fractal image compressing method [4] has received considerable attention since several years ago. This method has advantages of high compression ratio and resolution-independence. However, this method requires many processing times. So in this research, we focus on periodical images such as checked images seen in the textile design. And we propose a new image compressing method for checked textile design using the fast Fourier transform (FFT) [5] as the preprocessing method.

The remainder of this paper is organized as follows. In Section 2 , color space we apply in this research is presented. Concepts of compressing and decompressing methods in this proposed method are presented in Section 3 and 4, respectively. The results of applying this method to checked images are presented in Section 5. Finally, conclusions and a direction of future work are discussed in Section 6.

\section{Color space}

In order to obtain a value consistent with human color perception, the uniform lightness-chromaticness scale

\footnotetext{
* Corresponding author Fax:+81-792-67-4975, E-mail: ohta@comp.eng.himeji-tech.ac.jp
} 
(ULCS) system must be introduced as the color coordinate system. In this research, the $\mathrm{L}^{*} \mathrm{a}^{*} \mathrm{~b}^{*}$ color coordinate system [6] established by the Commission Internationale de l'Eclairage (CIE) is used as the standard ULCS color coordinate system. In order to convert from RGB color coordinate system to the $\mathrm{XYZ}$ color coordinate system, the following equations are used:

$$
\begin{aligned}
& X=0.49000 R+0.31000 G+0.20000 B \\
& Y=0.17697 R+0.81240 G+0.01063 B \\
& Z=0.01000 G+0.99000 B
\end{aligned}
$$

And the following equations are used to convert from the $\mathrm{XYZ}$ color coordinate system to the $\mathrm{L}^{*} \mathrm{a}^{*} \mathrm{~b}^{*}$ color coordinate system:

$$
\begin{aligned}
\mathrm{L}^{*} & =116(\mathrm{Y} / \mathrm{Yn})^{1 / 3}-16 \quad \mathrm{Y} / \mathrm{Yn}>0.008856 \\
& =903.3(\mathrm{Y} / \mathrm{Yn}) \quad \mathrm{Y} / \mathrm{Yn} \leqq 0.008856 \\
\mathrm{a}^{*} & =500\left[(\mathrm{X} / \mathrm{Xn})^{1 / 3}-(\mathrm{Y} / \mathrm{Yn})^{1 / 3}\right] \\
\mathrm{b}^{*} & =200\left[(\mathrm{Y} / \mathrm{Yn})^{1 / 3}-(\mathrm{Z} / \mathrm{Zn})^{1 / 3}\right]
\end{aligned}
$$

, where $\mathrm{Xn}, \mathrm{Yn}$, and $\mathrm{Zn}$ are the tristimulus values in the $\mathrm{XYZ}$ color coordinate system for a perfectly reflecting diffuse surface.

\section{Compressing method}

Checked images have many repeated patterns. Therefore, distributions in the spatial frequency domains are partially concentrated. A power spectrum figure of a sample checked image is shown in figure 1, and a part of distributions around local maximums is shown in figure 2 . In figure 2, power spectrum values are shown with logarithms, and normalized from 0 to 255 . In particular, the placement of different colored warp and weft yarn forms patterns on the textile surface in checked textile. Therefore, the warp and weft features are always continuous in the warp and weft directions [7]. By this research, it is clear to be able to obtain a reconstructed image close to an original image by using only data on the vertical and horizontal axes in the spatial frequency domains. As you can see in figure 2, each distribution around the local values can be approximately regarded as the normal distribution. So in this research, we focus on reconstructing only distributions on the vertical and horizontal axes in the spatial frequency domains to compress checked images. In order to reconstruct distributions on the vertical and horizontal axes in the spatial frequency domains, local values and characteristic values of distributions around the local values are used.

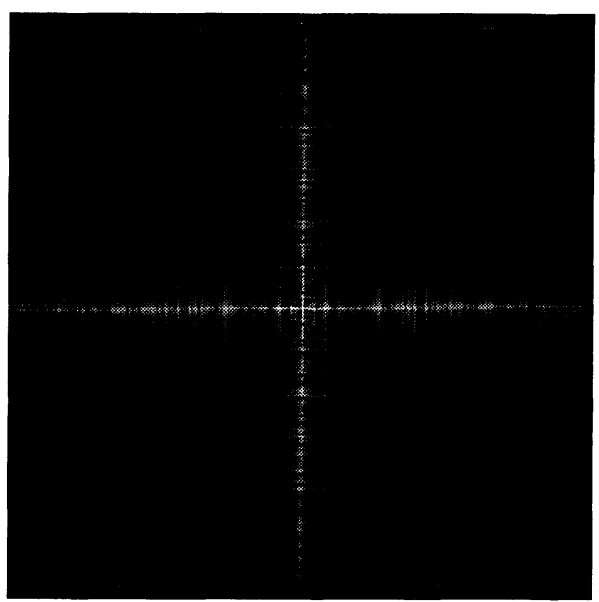

Fig. 1 A power spectrum figure of a checked image ( $255 \times 255$ )

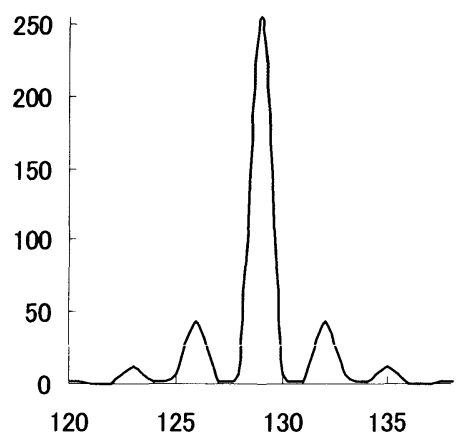

Fig. 2 A part of distributions around local maximums in Fig.1.

First, original images are obtained by the color image scanner and the images are converted from RGB color system to $\mathrm{L}^{*} \mathrm{a}^{*} \mathrm{~b}^{*}$ color system. Secondly, we apply FFT to data of each domain of $\mathrm{L}^{*}, \mathrm{a}^{*}$ and $\mathrm{b}^{*}$. We search positions that have local values on the vertical and horizontal axes in each spatial frequency domain of $\mathrm{L}, \mathrm{a}^{*}$, and $b^{*}$. And distributive shapes around the local values are investigated. We use four data as characteristics of the distributive shapes. Their four data are the mean, variance, skewness, and kurtosis value. The mean value is a position 
in the spatial frequency domains of having local values in this research. These four data are stored as codes with the local values. Finally, we use only these data as the compressed codes, and the other data aren't required. So, considerable higher compression ratio is expected. The procedure of compressing method is shown in figure 3 .

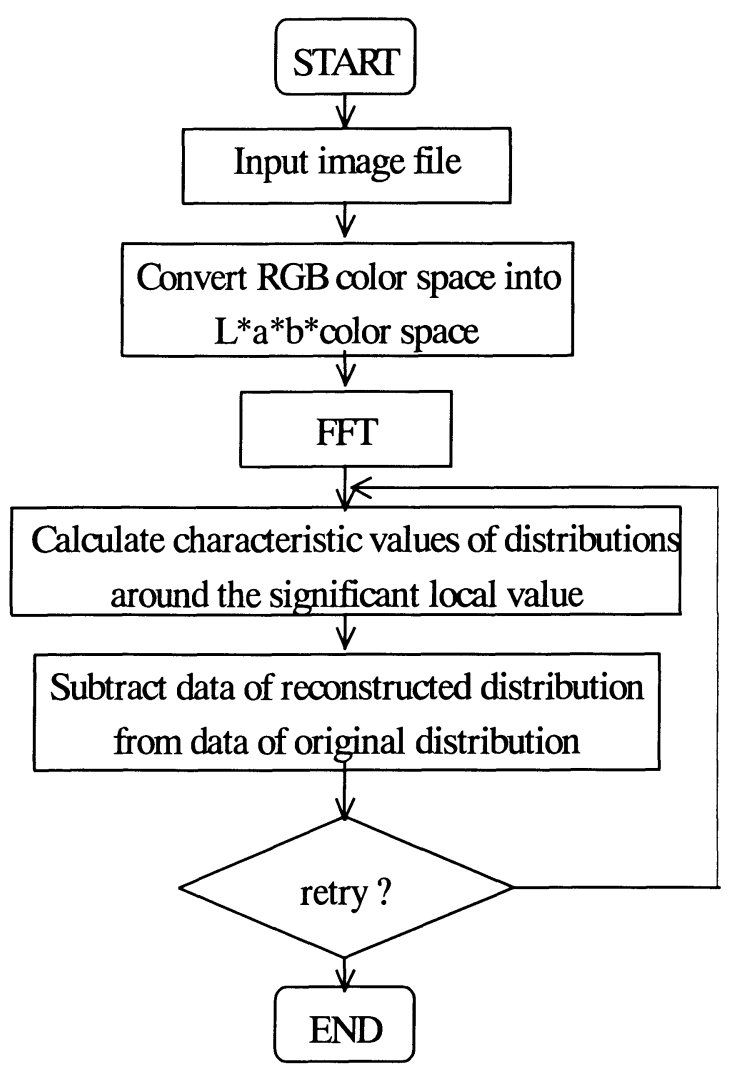

Fig. 3 The procedure of compressing method

\section{Decompressing method}

We reconstruct data on the vertical and horizontal axes in spatial frequency domains of $L^{*}, a^{*}$, and $b^{*}$ from the coded data. In this research, we utilize the approximate equation called Gram-Charlier series of Type A [2-3]. This function $f(x)$ is shown in equation (7).

$$
\begin{aligned}
f(x)= & \phi(x)\left\{1+\mu_{1} H_{1}(x)+\frac{\left(\mu_{2}-1\right)}{2} H_{2}(x)+\right. \\
& \left.\frac{\left(\mu_{3}-3 \mu_{1}\right)}{6} H_{3}(x)+\frac{\left(\mu_{4}-6 \mu_{2}+3\right)}{24} H_{4}(x)\right\}
\end{aligned}
$$

Where

$$
\phi(x)=\frac{1}{\sqrt{2 \pi} \sigma} \exp \left(-\frac{x^{2}}{2 \sigma^{2}}\right)
$$

$\mu_{r}$ is called the r-dimensional moment of the population.

And $H_{r}(x)$ is called Chebyshev-Hermite polynomials, and the following four equations are used in this research:

$$
\begin{aligned}
& H_{1}(x)=x \\
& H_{2}(x)=x^{2}-1 \\
& H_{3}(x)=x^{3}-3 x \\
& H_{4}(x)=x^{4}-6 x^{2}+3
\end{aligned}
$$

The reconstructed spatial frequency domains are obtained by the equation (7). We can obtain the reconstructed domains of $\mathrm{L}^{*}, \mathrm{a}^{*}$ and $\mathrm{b}^{*}$ by applying the inverse fast Fourier transform (IFFT) to the reconstructed spatial frequency domains. Finally, we can obtain the reconstructed images by converting $L^{*} a^{*} b^{*}$ color system into RGB color system. The procedure of decompressing method is shown in figure 4 .

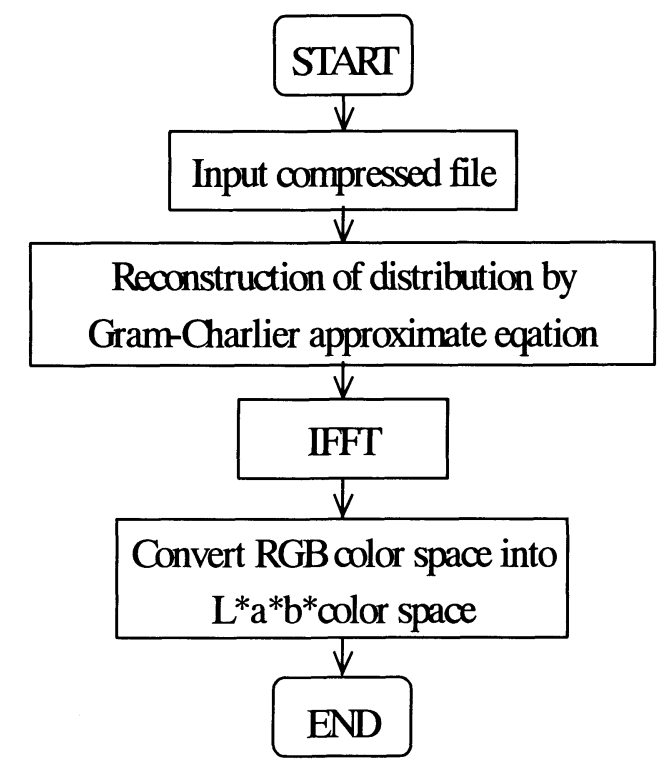

Fig. 4 The procedure of decompressing method

\section{Results}

In this research, we used three sample images. These images and the reconstructed images are shown in figure 5. As seen in figure 5, the patterns of the warp and weft of the 
original images are clearly reconstructed.

The compression ratio of this method depends on numbers of local values of distributions in the spatial frequency domains and size of images. So in order to compare the compression ratio of this method with that of JPEG format, sample image A is compressed by JPEG format. The compression ratios of this method and JPEG format are shown in table 1. Here, their signal to noise ratios are same. Table 1 shows that the compression ratio of this method is higher than that of JPEG format. However, the signal to noise ratio was low, because minute features cannot be obtained by only investigating distributions around the local values. However, the signal to noise is not very important in this research, because we would like to largely obtain information of the warp and weft in case of checked images.
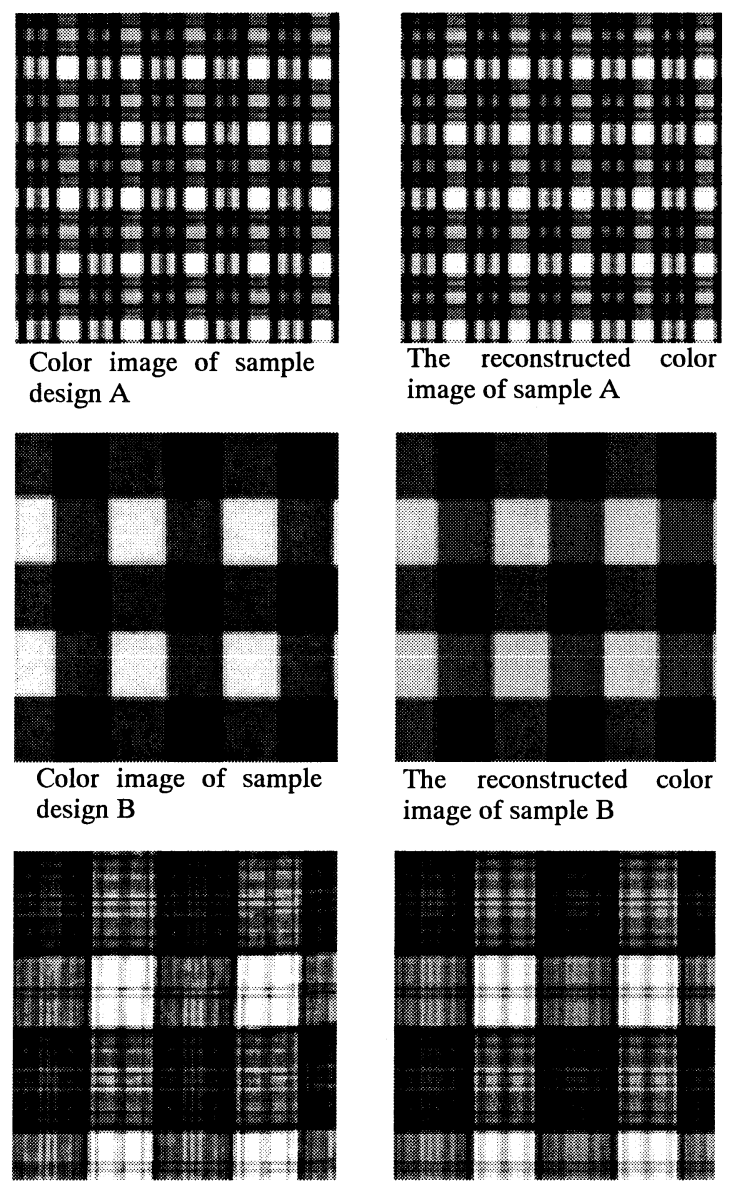

Color image of sample design $\mathrm{C}$

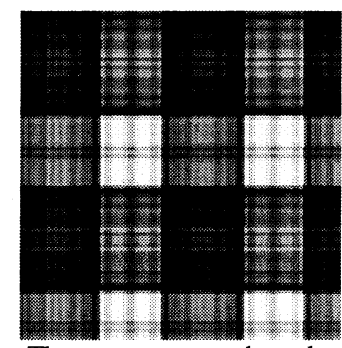

The reconstructed color image of sample $\mathrm{C}$

Fig. 5 Sample images and the reconstructed images

Table 1 Compression ratios of JPEG format and this proposed method

\begin{tabular}{c|c}
\hline Compressing method & Compression ratio (bytes) \\
\hline JPEG & $3145728: 33492$ \\
Proposed method & $3145728: 9628$ \\
\hline
\end{tabular}

\section{Conclusions}

In checked textile, the placement of different colored warp and weft yarn forms patterns on the textile surfaces. Therefore, the warp and weft features are always continuous in the warp and weft directions. So, we proposed the new image compressing method for checked textile design using this property. In this method, local values on the vertical and horizontal axes in the spatial frequency domains and characteristic values of distributions around the local values are used for compressed codes. Consequently, this method was quite effective for periodical images such as checked images. However, in case local values in the spatial frequency domains are near each other, distributions around the local values are overlapped each other, and it is difficult to clarify the characteristics. The examination of this point is a topic we would like to study in our future work.

\section{References}

[1] Nobuo Inao ; "Mathematical statistics", Syokabo Publisher (1990)

[2] Alan Stuart and J. Keith Ord ; "Kendall's Advanced Theory of Statistics, Volume 1 Distribution Theory", Edward Arnold (1994)

[3] Yoshisada Sibata ; "Normal Distribution", University of Tokyo Press (1981)

[4] Michael F. Barnsley and Lyman P. Hurd ; "Fractal image compression", A K Peters, Ltd. (1992)

[5] Syogo Nakamura ; "Digital Fourier transform", Tokyo Denki University Publisher (1989)

[6] Mikio Takagi and Haruhisa Simoda ; "Handbook of image analysis", University of Tokyo Press (1991)

[7] Ken'ich Ohta ; Use of Fourier Transform and Color Image Processing to Extract Design Elements of Yarn-Dyed Fabric, System and Computers in Japan, 24(9), 69-78 (1993) 